\title{
Left ventricular global longitudinal strain in low cardiac risk outpatients who recently recovered from coronavirus disease 2019
}

\author{
Turhan Turan $^{1}$ (1) - Ahmet Özderya ${ }^{1} \cdot$ Sinan Şahin ${ }^{1} \cdot$ Ali Hakan Konuş $^{1} \cdot$ Selim Kul ${ }^{1} \cdot$ Ali Rıza Akyüz $^{1}$. \\ Ezgi Kalaycıoğlu ${ }^{1} \cdot$ Muhammet Raşit Sayın ${ }^{1}$
}

Received: 20 June 2021 / Accepted: 4 August 2021 / Published online: 13 August 2021

(c) The Author(s), under exclusive licence to Springer Nature B.V. 2021

\begin{abstract}
The cardiac sequelae of coronavirus disease 2019 (COVID-19), a worldwide global pandemic, are still uncertain, particularly in the asymptomatic, low cardiac risk outpatient population. This study aims to evaluate the asymptomatic, low cardiac risk out-patient population who recently recovered from COVID-19, using 2-D left ventricular-global longitudinal strain (LV-GLS) proven to be capable of detecting subclinical myocardial injury. Out of 305 COVID-19 positive patients, 70 asymptomatic out-patients were determined as the study group and 70 age and sex-matched healthy adults as the control group. The echocardiographic examination was performed with the Philips IE33 system, and LV-GLS was measured using commercially available software QLAB 9 (cardiac motion quantification; Philips Medical Systems). The absolute value of LV-GLS $\leq 18$ did deem to be impaired LV-GLS. The absolute value of LV-GLS was statistically significantly lower in the COVID-19 group than in healthy controls $(19.17 \pm 2.65$ vs. $20.07 \pm 2.19, p=0.03)$. The correlation between having recovered from COVID-19 and impaired LV-GLS $(\leq 18)$ did detect with the Pearson correlation test $(p=0.02)$. Having recovered from COVID-19 was found as a predictor for detecting impaired LV-GLS $(\leq 18)$ in the multivariable logistic regression analysis (odds ratio, $0.133(0.038-0.461)$; 95\% CI, $p=0.001)$. This study suggests that COVID-19 may cause subclinical LV dysfunction detected by LV-GLS during early recovery even in a population of patients at low cardiac risk, asymptomatic, and recovered with home quarantine. The study findings indicate that the long-term cardiovascular follow-up of these patients may be more important than thought.
\end{abstract}

Keywords SARS coronavirus · Ventricular global longitudinal strain · Low cardiac risk · Home quarantine

\section{Introduction}

Ever since it was first identified in November 2019 and declared a global pandemic in 2020, coronavirus disease 2019 (COVID-19) remains one of the most common causes of mortality and morbidity, without losing popularity worldwide [1]. While the respiratory system is the main target of the Severe Acute Respiratory Syndrome Coronavirus-2 (SARS-CoV-2), which causes COVID-19, cardiovascular involvement, including myocarditis and cardiomyopathy, various arrhythmias, and heart failure, is increasingly being

Turhan Turan

drtt61@gmail.com

1 Department of Cardiology, University of Health Sciences Trabzon Ahi Evren Thoracic and Cardiovascular Surgery Training and Research Hospital, Vatan St. Ortahisar, Trabzon 61000, Turkey reported [2,3]. Also, many studies have shown that the mortality rate from COVID-19 increased more than three times in patients with underlying cardiovascular disease [4-6]. The main parameters used to determine COVID-19-induced cardiac injury in clinical practice are increased serum troponin levels and an overt reduction in left ventricular ejection fraction (LVEF) on the transthoracic echocardiography (ECHO) and reported in $8 \%$ to $28 \%$ of patients $[7,8]$. However, in recent studies using more sensitive diagnostic methods that can detect subclinical myocardial damage, such as cardiac magnetic resonance imaging (MRI) and 2-D left ventricular-global longitudinal strain (LV-GLS), reported cardiac involvement reached $80 \%[9,10]$.

Since the disease is a pandemic that maintains its popularity worldwide, the number of asymptomatic patients recovered from COVID-19 who apply to the cardiology outpatient clinic with the concern of cardiac sequelae is gradual. Data on cardiovascular sequelae associated with COVID-19 are 
insufficient, particularly in patients with normal ECG and cardiac markers, no cardiac symptoms, low to moderate cardiac risk, and recovered from the disease without hospitalization. The purpose of our study is to evaluate this low to moderate cardiac risk patient population who recently recovered from COVID-19 with home quarantine, using LV-GLS, which has proven to be able to detect subclinical myocardial injury without overt reduction in LVEF [11, 12].

\section{Materials and methods}

\section{Study design and population}

This study is a single-center, cross-sectional, prospective research held between December 2020 and March 2021 in Ahi Evren Thoracic and Cardiovascular Centre, Trabzon, Turkey. The study participants were prospectively enrolled from a total of 305 outpatients diagnosed with SARS-CoV-2 by reverse transcription-polymerase chain reaction (PCR) in the upper respiratory tract swab test and recently recovered from COVID-19 with ten days of home quarantine. The median (interquartile range) time interval between the initial diagnosis of COVID-19 and cardiology outpatient clinic examination was 23 (11-89) days. At the time of the first diagnosis of COVID-19 or admission to the cardiology outpatient clinic after the recovery, those who have lung involvement on computerized tomography or chest $\mathrm{X}$-ray, those with any cardiac symptoms (chest pain, syncope, palpitations, shortness of breath during ordinary daily activities), those with abnormal ECG (ST-segment elevation or $\geq 1 \mathrm{~mm}$ depression, any arrhythmia) and abnormal cardiac troponin I/T (cTnI/cTnT) values, were excluded. Besides, patients with older age (over 80 years old), low LVEF $(<50 \%)$, severe valvular heart disease, pre-existing CVD, pulmonary hypertension (PHT) $\geq$ stage 2 hypertension (HT), chronic obstructive pulmonary disease, malignancy, chronic liver or kidney disease [glomerular filtration rate (GFR) $<30 \mathrm{ml} / \mathrm{min}$ ] and poor echogenicity also excluded from the study. All participants above 40 years old did select from patients with low to moderate cardiac risk based on the Systemic Coronary Risk Assessment (SCORE) risk score (calculated SCORE <5\%) [13]. Among subjects under the age of 40 , with a family history of early cardiovascular disease (CVD) in a first-degree relative (sibling or parent), documented familial hypercholesterolemia, juvenile-onset

Patients who recently recovered from COVID - 19 throught home quarantine ( $\mathrm{n}=305)$

\section{Inclusion criteria:}

At the time of the first diagnosis of COVID-19 or admission to the cardiology outpatient clinic after the recovery without any of the following:

- lug involvement on computerized tomography or chest X-ray

- any cardiac symptoms (chest pain, syncope, palpitations, shortness of breath during ordinary daily activities)

- abnormal ECG (ST-segment elevation or $1 \mathrm{~mm}$ depression, any arrythmia)

- abnormal cardiac high-sensitivity troponin I/T (hs-TnI / TnT) values

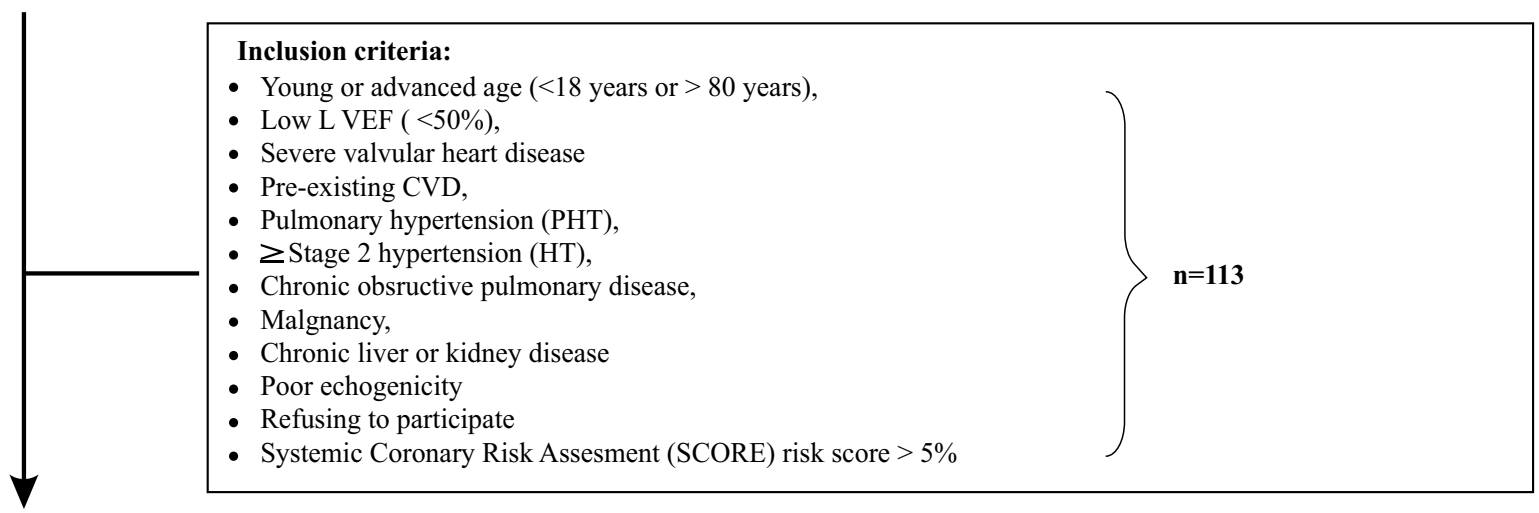

Final study cohort $(\mathrm{n}=70)$

Fig. 1 Flowchart of the study 
type 1 diabetes (DM), or early-onset type $2 \mathrm{DM}$ and multiple cardiovascular risk factors patients excluded. The study patients were selected from asymptomatic individuals who applied to the cardiology outpatient clinic with the concern about cardiac sequelae after the recovery from COVID-19 (patients; $n=70$ ) (Fig. 1). According to SCORE, the control group consisted of age- and sex-matched adult subjects with low to moderate cardiac risk (healthy controls; $n=70$ ). The study protocol did approve by the local Ethics Committee of the University of Health Sciences Trabzon Kanuni Training and Research Hospital and the Republic of Turkey Ministry of Health. The study was conducted in concordance with the Helsinki Declaration and the International Conference on Good Clinical Practices Harmony, and written informed consent did obtain from all participants.

Laboratory and clinical information at the initial diagnosis were collected from electronic medical records and repeated at admission to the cardiology outpatient clinic. Clinical, demographic characteristics, medications were re-inquired, blood tests, chest X-ray, and ECG repeated. Venous blood samples were obtained just before the ECHO examination. Biochemistry measurements, including liver and renal function tests, total cholesterol, low-density lipoprotein (LDL), high-density lipoprotein (HDL), triglycerides (TG), high-sensitivity troponin I (hs-TnI), C-reactive protein (CRP), did perform. Hematological parameters were measured as part of the automated complete blood count (CBC) using a Mindray BC-5800 auto hematology analyzer (Mindray Medical Electronics Co. Shenzhen, China). Hypertension diagnosis and stratification did base on recent guidelines [14]. Hypercholesterolemia did define as the total cholesterol $>200 \mathrm{mg} / \mathrm{dl}$. Estimating GFR was calculated by using Cockcroft-Gault (CG) formula. [15].

\section{Echocardiographic evaluation}

The echocardiographic examination was performed with the Philips IE33 system (Philips Medical Systems, Andover, MA, USA). Standardized transthoracic and Doppler echocardiographic examinations were performed under continuous electrocardiographic recording. Data acquisition was completed by a 5-1 MHz sector transducer in the parasternal and apical views (standard 2- and 4-chamber views). All echocardiograms were read off-line by two experienced cardiologists blind to patient status in line with the American Society of Echocardiography (ASE) recommendations [16]. Two-dimensional and Doppler images were obtained during breath-hold and stored in cine-loop format from 3 consecutive beats, and average values were reported. The left ventricle (LV) end-systolic volume (ESV), end-diastolic volume (EDV), and ejection fraction (EF) were derived by the apical biplane modified Simpson rule. Doppler measurements included peak early mitral filling velocity (E wave), peak late mitral filling velocity (A wave), the ratio of peak early and late mitral filling velocities (E/A). Tricuspid annular plane systolic excursion (TAPSE) was recorded on M-mode at the junction between the right ventricular lateral wall and tricuspid annulus in apical 4-chamber view. For myocardial tissue velocities, tissue Doppler imaging (TDI) sample volume was placed at the lateral mitral and tricuspid annulus, at the junction between the left and right ventricular lateral wall and mitral and tricuspid annulus, in apical 4-chamber view. Tricuspid lateral annular systolic velocity (S') assessed by tissue Doppler imaging from the apical 4 -chamber view at the lateral segment of the right ventricle. TDI also included the following parameters; early diastolic myocardial velocity (E'), late diastolic myocardial velocity (A'), and E'/A'. Also, E/E' was used to evaluate estimated left ventricular end-diastolic pressure. According to the update from the American Society of Echocardiography and the European Association of Cardiovascular Imaging (EACI), diastolic dysfunction was graded according to the update from the American Society of Echocardiography [17]. LVGLS was measured using the Philips IE33 system (Philips Medical Systems, Andover, MA, USA) by commercially available software QLAB 9 (cardiac motion quantification; Philips Medical Systems) (Fig. 2). Endocardial borders did monitor within the frame of $2 \mathrm{D}$ images at the end of the systole. Automatic endocardial contour tracking was performed in end-systole by a software (analysis) program, or manual adjustment was performed if necessary. The timing of end-diastole was determined by the peak $\mathrm{R}$ wave of the electrocardiogram, while the timing of end-systole was determined by aortic valve closure. The analysis of LV-GLS was performed from the three apical views (four-, three-, and two-chamber). The mean longitudinal strain was calculated by averaging the peak longitudinal strain values of three apical views. The reference LV-GLS limit expressed as a negative value is $<-18 \%$. Namely, the more negative values indicate better cardiac function. Absolute values of LVGLS were considered as $>18$ reference, 16-18 Gray zones, and $<16$ decreased. All values 18 and below did deem to be impaired LV-GLS.

\section{Statistical analysis}

SPSS 21.0 for Windows (SPSS Inc., Chicago, IL, USA) did use for statistical analyses. Quantitative data expressed as mean \pm standard deviation (SD). Categorical data were presented as number (n) and frequency (\%). For the suitable analysis technique to choose, Kolmogorov-Smirnov, and homogeneity of variance tests were undertaken. Independent samples t-test used for the two-group comparison of the normally distributed variables and Mann-Whitney U-test for the two-group comparison of the variables without normal 
Fig. 2 Left ventricle global longitudinal strain; A Regional strain imaging in apical four chambers; B Regional strain imaging in apical two chambers; C Regional strain imaging in apical three chambers; D Global longitudinal peak systolic strain imaging

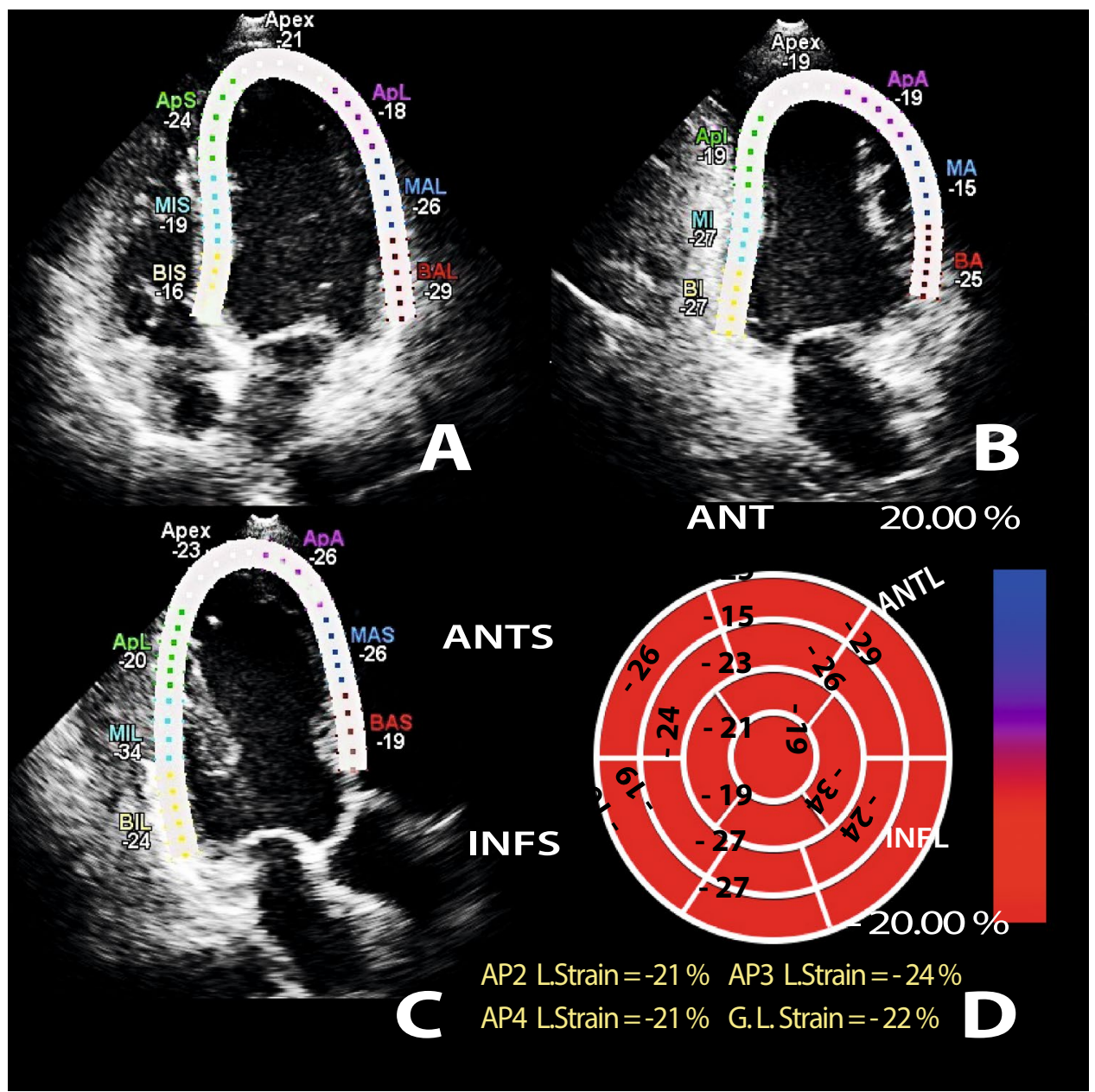

distribution. Categorical variables were compared using the Chi-square test. Pearson or Spearman correlation analysis were used for determining associations, as appropriate for the type of data. The independent predictors of impaired LV function (LV-GLS $\geq-18$ or absolute $\leq 18$ ) were calculated using multivariable logistic regression analysis. Took the following clinical variables into account in the multivariate procedure: age, gender, history of hypertension, smoking and asthma, body mass index, glomerular filtration rate, serum LDL-C, CRP and Hb levels, E/E', diastolic dysfunction, and to have recovered from COVID-19. Assessed intraand inter-observer variability analysis of LV-GLS data by reanalyzing 15 randomly selected patients (intra-observer reliability) and calculating from a second independent observer (inter-observer reproducibility). The compatibility of intra- and inter-observer LV-GLS had calculated, and the correlation coefficient of variation and intra- class correlations had evaluated. A $p<0.05$ was considered statistically significant.

\section{Results}

In this study, a total of 305 patients who recently recovered from COVID-19 infection has screened. After the exclusion criteria, the remaining 70 patients ( 37 women and 33 men; the mean age was $43.59 \pm 11.83$ years) has determined as the study group. The control group consisted of 70 (34 women and 36 men; the mean age was $46.66 \pm 16.58$ years) age- and sex-matched healthy adults. Short-term medical treatments of COVID-19 patients included favipiravir $88 \%$, hydroxychloroquine $18 \%$, paracetamol $78 \%$, low molecular weight heparin $14 \%$, acetylsalicylic acid $70 \%$, and vitamin C 68\%, in line with our national health practice. The median (interquartile range) time duration of medical treatment was 5 (3-7) days. Baseline characteristics of the subjects had provided in Table 1. Basal characteristics of the groups were similar, except for the serum levels of hemoglobin, hematocrit, mean corpuscular volume (MCV), and albumin. Plasma hemoglobin, hematocrit, and MCV levels were lower, and the albumin level was higher in the COVID-19 group than in the controls $(13.58 \pm 1.59 \mathrm{~g} / \mathrm{dL}$ vs. $14.37 \pm 1.43 \mathrm{~g} /$ $\mathrm{dL}, 40.02 \pm 4.15 \%$ vs. $42.26 \pm 4.24 \%, 85.92 \pm 4.07 \mathrm{fl}$ 
Table 1 Baseline clinical characteristics of the study population

\begin{tabular}{|c|c|c|c|}
\hline Characteristics & COVID-19 group $(n=70)$ & Healthy Controls $(n=70)$ & $P$ \\
\hline Age (years) & $43.59 \pm 11.83$ & $46.66 \pm 16.58$ & $0.209^{2}$ \\
\hline Sex (male) $(n)(\%)$ & $33(47.1)$ & $36(51.4)$ & $0.612^{\mathrm{b}}$ \\
\hline $\operatorname{BMI}\left(\mathrm{kg} / \mathrm{m}^{2}\right)$ & $27.52 \pm 4.48$ & $27.80 \pm 5.32$ & $0.735^{\circ}$ \\
\hline Hypertension, $n(\%)$ & $9(12)$ & $12(17)$ & 0.478 \\
\hline Smoking, $n(\%)$ & $12(17)$ & $18(26)$ & $0.217^{\mathrm{b}}$ \\
\hline Alcohol addiction, $n(\%)$ & $0(0)$ & $2(3)$ & $0.154^{\mathrm{b}}$ \\
\hline Asthma, $n(\%)$ & $8(11)$ & $4(6)$ & $0.227^{\mathrm{t}}$ \\
\hline Hemoglobin $(\mathrm{g} / \mathrm{dL})$ & $13.58 \pm 1.59$ & $14.37 \pm 1.43$ & $0.003^{\circ}$ \\
\hline Hematocrit (\%) & $40.02 \pm 4.15$ & $42.26 \pm 4.24$ & $0.002^{2}$ \\
\hline $\operatorname{MCV}(\mathrm{fl})$ & $85.92 \pm 4.07$ & $87.61 \pm 4.50$ & 0.022 \\
\hline WBC $\left(\times 10^{9} / \mathrm{L}\right)$ & $7.49 \pm 1.61$ & $7.42 \pm 1.94$ & $0.839^{\circ}$ \\
\hline $\operatorname{PLT}\left(\times 10^{9} / \mathrm{L}\right)$ & $243.42 \pm 65.54$ & $233.65 \pm 46.47$ & $0.312^{2}$ \\
\hline MPV (fl) & $8.37 \pm 1.53$ & $8.32 \pm 1.18$ & $0.826^{\circ}$ \\
\hline Creatinine $(\mathrm{mg} / \mathrm{dL})$ & $0.84 \pm 0.18$ & $0.81 \pm 0.11$ & $0.236^{\circ}$ \\
\hline GFR (ml/min.) & $98.48 \pm 16.20$ & $97.70 \pm 15.87$ & $0.775^{\circ}$ \\
\hline Urea (mg/dL) & $28.65 \pm 8.20$ & $29.20 \pm 9.69$ & 0.720 \\
\hline Glucose $(\mathrm{mg} / \mathrm{dL})^{\#}$ & $95(68-187)$ & $95(73-254)$ & $0.284^{\circ}$ \\
\hline Sodium $(\mathrm{mmol} / \mathrm{L})$ & $140.43 \pm 2.29$ & $140.25 \pm 1.43$ & $0.583^{\circ}$ \\
\hline Potassium $(\mathrm{mmol} / \mathrm{L})$ & $4.27 \pm 0.37$ & $4.32 \pm 0.33$ & $0.432^{\circ}$ \\
\hline Calcium (mmol/L) & $9.17 \pm 0.35$ & $9.22 \pm 0.42$ & 0.574 \\
\hline $\operatorname{ALT}(\mathrm{U} / \mathrm{L})^{\#}$ & $23(10-201)$ & $20(7-62)$ & $0.192^{c}$ \\
\hline $\operatorname{AST}(\mathrm{U} / \mathrm{L})^{\#}$ & $20(12-65)$ & $20(8-54)$ & $0.959^{\circ}$ \\
\hline Total protein $(\mathrm{g} / \mathrm{L})$ & $74.04 \pm 3.67$ & $72.35 \pm 5.69$ & $0.230^{2}$ \\
\hline Albumin $(\mathrm{g} / \mathrm{L})$ & $43.71 \pm 2.70$ & $42.23 \pm 2.42$ & $0.040^{\circ}$ \\
\hline $\mathrm{CRP}(\mathrm{mg} / \mathrm{dL})^{\#}$ & $0.5(0.3-42)$ & $0.5(0.1-17)$ & $0.204^{c}$ \\
\hline LDL-C (mg/dL) & $127.11 \pm 39.15$ & $127.29 \pm 39.87$ & $0.981^{\circ}$ \\
\hline HDL-C (mg/dL) & $51.35 \pm 11.93$ & $52.66 \pm 15.15$ & $0.725^{\circ}$ \\
\hline Total Cholesterol (mg/dL) & $189.54 \pm 28.68$ & $183.53 \pm 49.58$ & 0.604 \\
\hline Triglyceride $(\mathrm{mg} / \mathrm{dL})^{\#}$ & $138(46-542)$ & 139 (89-207) & $0.906^{c}$ \\
\hline hs- Troponin I (ng/L) & $0.65(0.1-7.9)$ & $1.4(0.1-6.78)$ & $0.073^{c}$ \\
\hline SCORE, $\%^{\#}$ & $0.0(0-4)$ & $0.5(0-4)$ & $0.494^{\circ}$ \\
\hline \multicolumn{4}{|l|}{ Drug Using } \\
\hline Statin, $n,(\%)$ & $11(15.7)$ & $5(7.1)$ & $0.183^{\mathrm{b}}$ \\
\hline$A C E I / A R B, n,(\%)$ & $7(10)$ & $9(12.9)$ & $0.791^{\mathrm{b}}$ \\
\hline Beta blocker, $n,(\%)$ & $1(1.4)$ & $1(1.4)$ & $1^{\mathrm{b}}$ \\
\hline Calcium channel blocker, $n,(\%)$ & $1(1.4)$ & $3(4.3)$ & $0.620^{\mathrm{b}}$ \\
\hline \multicolumn{4}{|l|}{ Temporary COVID-19 treatments ${ }^{*}$} \\
\hline Favipravir $n,(\%)$ & $62(88)$ & & \\
\hline Hydroxychloroquine $n,(\%)$ & $13(18)$ & & \\
\hline Paracetamol $n,(\%)$ & $55(78)$ & & \\
\hline$L M W H n,(\%)$ & $10(14)$ & & \\
\hline Salicylic Acid (Aspirin) $n,(\%)$ & $49(70)$ & & \\
\hline Vitamin $C n,(\%)$ & $48(68)$ & & \\
\hline
\end{tabular}

$A C E I / A R B$ Angiotensin converting enzyme inhibitor/angiotensin receptor blocker, ALT Alanine amino transferase, AST Aspartate amino transferase, BMI Body mass index, $C R P$ C-reactive protein, GFR Glomerular filtration rate, $H D L-C$ High density lipoprotein cholesterol, $h s$-Troponin I High-sensitivity troponin I, $I Q R$ Interquartile range, $L D L-C$ Low density lipoprotein cholesterol, $L M W H$ Low molecular weight heparin, $M C V$ Mean corpuscular volume, MPV Mean platelet volume, PLT Platelet, SCORE Systemic coronary risk assessment risk score, $W B C$ White blood cell

${ }^{\mathrm{a}}$ Independent $\mathrm{t}$ test, ${ }^{\mathrm{b}} \mathrm{Chi}$-square test, ${ }^{\mathrm{c}} \mathrm{Mann}-$ Whitney U test. ${ }^{\#}$ Data are expressed as median (IQR) for continuous variables. ${ }^{*}$ [median 5 (IQR 3-7) days] 
Table 2 Comparison of the echocardiographic parameters of patients and controls

\begin{tabular}{|c|c|c|c|}
\hline ECHO parameters & COVID-19 group $(n=70)$ & Healthy Controls $(n=70)$ & $P$ \\
\hline LV-GLS (absolute value, \%) & $19.17 \pm 2.65$ & $20.07 \pm 2.19$ & $0.030^{\mathrm{a}}$ \\
\hline LV-EF (\%) & $64.89 \pm 3.99$ & $64.34 \pm 3.13$ & $0.328^{\mathrm{a}}$ \\
\hline LV-EDD (mm) & $44.71 \pm 4.56$ & $44.63 \pm 3.98$ & $0.906^{\mathrm{a}}$ \\
\hline LV-ESD (mm) & $27.86 \pm 4.04$ & $27.16 \pm 3.65$ & $0.285^{\mathrm{a}}$ \\
\hline LV-EDV (mL) & $92.45 \pm 21.66$ & $91.71 \pm 19.54$ & $0.833^{\mathrm{a}}$ \\
\hline LV-ESV $(m L)^{\#}$ & $27.02(11-62)$ & $27.01(13-58)$ & $0.362^{\mathrm{b}}$ \\
\hline LV-EDV index $\left(\mathrm{mm} / \mathrm{m}^{2}\right)$ & $24.17 \pm 2.66$ & $24.30 \pm 2.80$ & $0.779^{\mathrm{a}}$ \\
\hline LV-ESV index $\left(\mathrm{mm} / \mathrm{m}^{2}\right)$ & $15.05 \pm 2.20$ & $14.77 \pm 2.16$ & $0.441^{\mathrm{a}}$ \\
\hline FS $(\%)$ & $37.83 \pm 4.83$ & $39.27 \pm 4.69$ & $0.076^{\mathrm{a}}$ \\
\hline $\operatorname{IVS}(\mathrm{mm})^{\#}$ & $10(7-12)$ & $9(6-12)$ & $0.066^{\mathrm{b}}$ \\
\hline $\mathrm{PW}(\mathrm{mm})^{\#}$ & $9(7-12)$ & $9(6-12)$ & $0.189^{\mathrm{b}}$ \\
\hline $\mathrm{LA}(\mathrm{mm})$ & $32.44 \pm 4.5$ & $32.89 \pm 4.29$ & $0.553^{\mathrm{a}}$ \\
\hline $\mathrm{E}(\mathrm{cm} / \mathrm{s})$ & $78.47 \pm 19.48$ & $73.92 \pm 18.95$ & $0.164^{\mathrm{a}}$ \\
\hline $\mathrm{A}(\mathrm{cm} / \mathrm{s})$ & $69.50 \pm 15.53$ & $62.48 \pm 14.77$ & $0.007^{\mathrm{a}}$ \\
\hline$E^{\prime}(\mathrm{cm} / \mathrm{s})$ & $13.22 \pm 3.80$ & $12.47 \pm 3.97$ & $0.256^{\mathrm{a}}$ \\
\hline $\mathrm{A}^{\prime}(\mathrm{cm} / \mathrm{s})$ & $11.54 \pm 3.69$ & $10.18 \pm 3.32$ & $0.024^{\mathrm{a}}$ \\
\hline E/E' ratio (average) & $6.22 \pm 1.65$ & $6.29 \pm 1.84$ & $0.825^{\mathrm{a}}$ \\
\hline Diastolic Function, $n(\%)$ & & & $0.269^{\mathrm{c}}$ \\
\hline Normal & $37(52.9)$ & $44(62.9)$ & \\
\hline Grade 1 DD & $20(28.6)$ & $18(25.7)$ & \\
\hline Grade 2 DD & $10(14.3)$ & $8(11.4)$ & \\
\hline Grade 3 DD & $3(4.3)$ & $0(0)$ & \\
\hline Grade 4 DD & $0(0)$ & $0(0)$ & \\
\hline Mitral Regurgitation, $n(\%)$ & & & $0.791^{\mathrm{c}}$ \\
\hline None & $63(90)$ & $61(87.1)$ & \\
\hline Mild & $7(10)$ & $9(12.9)$ & \\
\hline Moderate & $0(0)$ & $0(0)$ & \\
\hline Severe & $0(0)$ & $0(0)$ & \\
\hline Aortic velocity $(\mathrm{cm} / \mathrm{s})$ & $135.39 \pm 21.86$ & $134.14 \pm 15.70$ & $0.700^{\mathrm{a}}$ \\
\hline $\mathrm{RA}^{\#}(\mathrm{~mm})$ & $32(24-42)$ & $30(23-39)$ & $0.600^{\mathrm{b}}$ \\
\hline $\mathrm{RV}(\mathrm{mm})$ & $27.59 \pm 3.52$ & $26.75 \pm 3.65$ & $0.171^{\mathrm{a}}$ \\
\hline TAPSE (mm) & $25.77 \pm 3.80$ & $24.84 \pm 3.42$ & $0.135^{\mathrm{a}}$ \\
\hline$S^{\prime}(\mathrm{cm} / \mathrm{s})$ & $13.88 \pm 2.33$ & $12.85 \pm 2.46$ & $0.013^{\mathrm{a}}$ \\
\hline
\end{tabular}

$A$ Peak late mitral filling velocity, $A^{\prime}$ Late diastolic myocardial velocity, $D D$ Diastolic dysfunction, $E$ Peak early mitral filling velocity, $E$ ' Early diastolic myocardial velocity, FS Fractional shortening, IQR Interquartile range, IVS Interventricular septum, $L A$ Left atrium, $L V$-EDD Left ventricular end-diastolic diameter, $L V$-EDV Left ventricular end-diastolic volume, $L V$-EF Left ventricular ejection fraction, $L V$ - $E S D$ Left ventricular end-systolic diameter, $L V$-ESV Left ventricular end-systolic volume, $L V$-GLS Left ventricular global longitudinal strain, $P W$ Posterior wall, $R A$ Right atrium, $R V$ Right ventricle, $S$ ' Tricuspid lateral annular systolic velocity, TAPSE Tricuspid annular plane systolic excursion

${ }^{\mathrm{a}}$ Independent $\mathrm{t}$ test, ${ }^{\mathrm{b}} \mathrm{Mann}-$ Whitney U test, ${ }^{\mathrm{c}}$ Chi-square test, ${ }^{\#}$ Data are expressed as median (IQR) for continuous variables vs. $87.61 \pm 4.50 \mathrm{fl}$, and $43.71 \pm 2.70 \mathrm{~g} / \mathrm{L}$ vs. $42.23 \pm 2.42 \mathrm{~g} / \mathrm{L}$; $p=0.003,0.002,0.02$, and 0.04 respectively).

The echocardiographic characteristics of the study groups had provided in Table 2. The median (interquartile range) time interval between the initial diagnosis of COVID-19 and ECHO examination was 23 (11-89) days. There was no significant correlation between ECHO parameters and time from the initial COVID-19 diagnosis in the patient group (LV-GLS: $\mathrm{r}=-0.006, p=0.96$, and also $p>0.05$ for all of the other classical ECHO parameters, too) (Table 3). There were no differences between the COVID-19 and control groups in terms of the classical ECHO parameters except the peak late mitral filling velocity (A wave), peak late diastolic myocardial velocity (A'), and tricuspid lateral annular systolic velocity (S'). A, A' and S' wave velocities were higher in the COVID-19 group $(69.50 \pm 15.53 \mathrm{~cm} / \mathrm{s}$ vs. 
Table 3 Relationship between echocardiographic parameters and time from initial COVID-19 diagnosis

\begin{tabular}{lcl}
\hline Parameter & $\begin{array}{l}\text { Correlation Coef- } \\
\text { ficient (r) }\end{array}$ & $P$ \\
\hline LV-GLS (absolute value), $\%$ & -0.006 & $0.962^{\mathrm{a}}$ \\
LV-EF (\%) & 0.034 & $0.777^{\mathrm{b}}$ \\
LV-EDD (mm) & 0.184 & $0.128^{\mathrm{b}}$ \\
LV-ESD (mm) & 0.123 & $0.309^{\mathrm{b}}$ \\
LV-EDV index $\left(\mathrm{mm} / \mathrm{m}^{2}\right)$ & 0.179 & $0.138^{\mathrm{b}}$ \\
LV-ESV index $\left(\mathrm{mm} / \mathrm{m}^{2}\right)$ & 0.086 & $0.479^{\mathrm{a}}$ \\
FS $(\%)$ & 0.007 & $0.955^{\mathrm{b}}$ \\
IVS (mm) & -0.071 & $0.557^{\mathrm{a}}$ \\
PW (mm) & 0.022 & $0.859^{\mathrm{a}}$ \\
LA (mm) & 0.147 & $0.225^{\mathrm{b}}$ \\
E (cm/s) & -0.021 & $0.866^{\mathrm{b}}$ \\
A (cm/s) & -0.071 & $0.561^{\mathrm{b}}$ \\
E' (cm/s) & -0.140 & $0.248^{\mathrm{b}}$ \\
A' (cm/s) & 0.011 & $0.930^{\mathrm{b}}$ \\
E/E'ratio (average) & 0.108 & $0.373^{\mathrm{b}}$ \\
Diastolic Dysfunction & 0.100 & $0.412^{\mathrm{b}}$ \\
Ascendant aorta diameter (mm) & -0.050 & $0.685^{\mathrm{a}}$ \\
Aortic velocity (cm/s) & -0.067 & $0.584^{\mathrm{b}}$ \\
RA $(\mathrm{mm})$ & -0.006 & $0.959^{\mathrm{a}}$ \\
RV (mm) & 0.152 & $0.211^{\mathrm{b}}$ \\
TAPSE (mm) & -0.049 & $0.687^{\mathrm{b}}$ \\
S' (cm/s) & 0.079 & $0.514^{\mathrm{b}}$ \\
\hline
\end{tabular}

$A$ Peak late mitral filling velocity, $A^{\prime}$ Late diastolic myocardial velocity, $E$ Peak early mitral filling velocity, $E$ ' Early diastolic myocardial velocity, $F S$ Fractional shortening, IQR Interquartile range, IVS Interventricular septum, $L A$ Left atrium, $L V$-EDD Left ventricular enddiastolic diameter, $L V$-EDV Left ventricular end-diastolic volume, $L V$-EF Left ventricular ejection fraction, $L V$-ESD Left ventricular end-systolic diameter, $L V$-ESV Left ventricular end-systolic volume, $L V$-GLS Left ventricular global longitudinal strain, $P W$ Posterior wall, $R A$ Right atrium, $R V$ Right ventricle, $S$ ' Tricuspid lateral annular systolic velocity, TAPSE Tricuspid annular plane systolic excursion

${ }^{\text {a }}$ Pearson correlation analysis, ${ }^{\text {b }}$ Spearman correlation analysis

$62.48 \pm 14.77 \mathrm{~cm} / \mathrm{s}, 11.54 \pm 3.69 \mathrm{~cm} / \mathrm{s}$ vs. $10.18 \pm 3.32 \mathrm{~cm} / \mathrm{s}$ and $13.88 \pm 2.33 \mathrm{~cm} / \mathrm{s}$ vs. $12.85 \pm 2.46 \mathrm{~cm} / \mathrm{s}, p=0.007,0.024$ and 0.013 respectively).

Inter- and intra-observer agreement for LV-GLS measurements revealed high agreement; intra-observer: 0.94 [95\% confidence interval (CI), 0.91-0.97], and interobserver: 0.91 (95\% CI 0.85-0.96). The absolute values of LV-GLS were statistically significantly lower in the COVID-19 group than in healthy controls $(19.17 \pm 2.65$ vs. $20.07 \pm 2.19, p=0.03)$. The correlation between having recovered from COVID-19 and impaired LV-GLS $(\leq 18)$ did detect with the Pearson correlation test $(p=0.02$, Correlation Coefficient: -0.197). In addition, the proportion of patients with impaired LV-GLS was statistically significantly higher in the COVID-19 group than in healthy controls when included the gray region of the LVGLS cut-off value (absolute value $\leq 18$ ) and not included (absolute value < 16) [30 (42.9\%) vs. $17(24.3 \%), p=0.02$, and $4(5.7 \%)$ vs. $0(0 \%), p=0.04$, respectively] (Fig. 3). In the multivariable logistic regression analysis, it had found that having recovered from COVID-19 was a predictor for detecting impaired LV-GLS (absolute value $\leq 18$ ) (odds ratio [OR], $0.133(0.038-0.461) ; 95 \% \mathrm{CI}, p=0.001)$. Age was the other predictor $(p=0.039)$ (Table 4).

\section{Discussion}

In this study, we found that asymptomatic patients who recovered from COVID-19 had decreased LV-GLS values than the healthy controls, irrespective of the duration of the first diagnosis. The number of patients with impaired LVGLS was statistically significantly higher in the COVID-19 group than the healthy controls. Also, logistic regression analysis showed that recovery from COVID-19 was a predictor for detecting impaired LV-GLS in our patient population.

To our knowledge, this is the first prospective study on a cohort of asymptomatic, low-intermediate cardiac risk patients a recently recovered from COVID-19 with home quarantine. The findings of our study provide essential information that COVID-19 may cause subclinical myocardial damage in the early recovery phase, even in such a low-risk, asymptomatic, and outpatient population. This suggests the importance of long-term clinical follow-up of these patients in terms of cardiology.

Overt cardiac dysfunction associated with COVID-19, defined by an increase in cTn and decreased LVEF, had been reported to range from $8 \%$ to $28 \%$ [7, 8]. However, later, it has been shown that subclinical cardiac involvement determined by more sensitive methods could be much higher. One of these methods, LV-GLS, has been validated for early detection of changes in subclinical left ventricular longitudinal function that precede overt LVEF impairment [18-20]. Moreover, it had proven to predict future cardiac events, including heart failure independent of LVEF, sex, age, and hypertension in large cohorts [21]. GLS is a simple parameter that expresses longitudinal shortening as a percentage and is calculated from all modern strain software. LV-GLS does not have a clear cut-off value and has a wide range of expected values due to vendor variability (type and model) of speckle tracking analysis. According to the most recent comprehensive reports in the literature for LV-GLS, the value of above $-18 \%$ did define as standard, $-16--18 \%$ as gray zone, under $-6 \%$ as diminished $[22,23]$. In addition, the latest expert consensus document of the European Association of Cardiovascular Imaging (EACVI) declared that a cut-off value upon $-18 \%$ (although magnitudes are vendor 


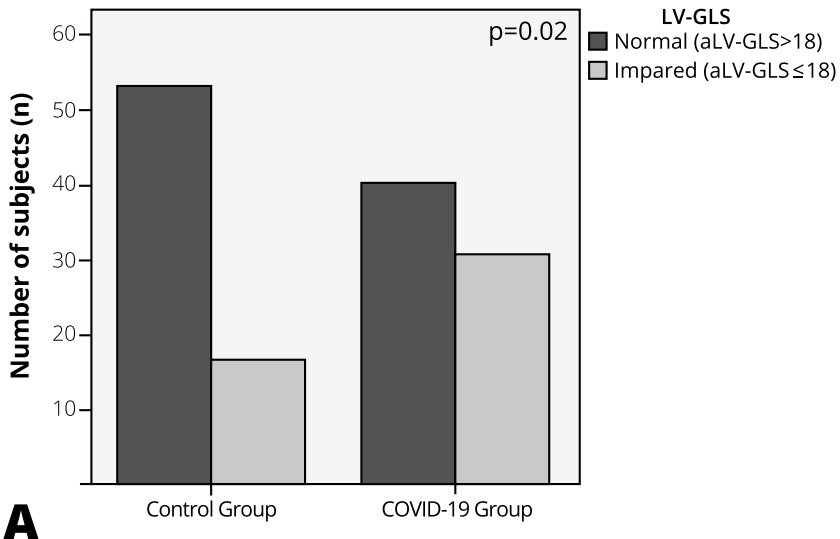

aLV-GLS: The absolute value of left ventricular global longitudinal strain (\%)

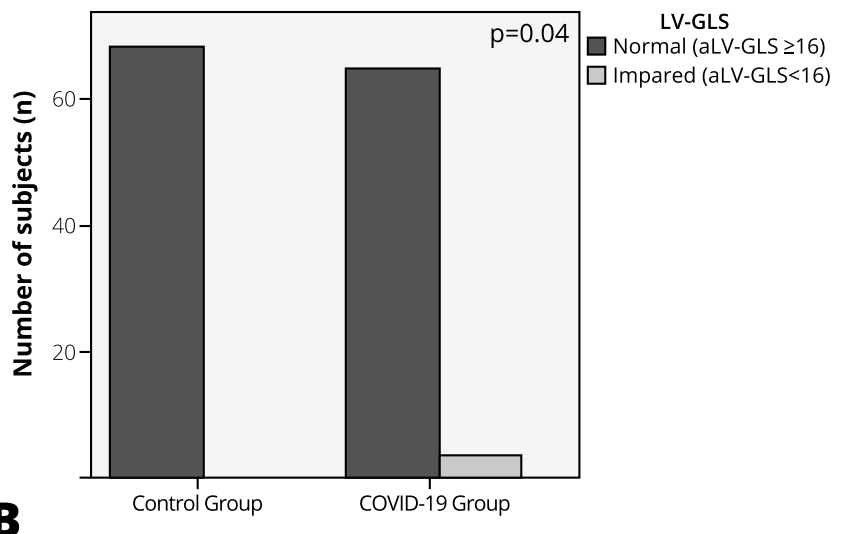

aLV-GLS: The absolute value of left ventricular global longitudinal strain (\%)

Fig. 3 Compare the number of patients with impaired left ventricular global longitudinal strain between groups $\mathbf{A}$ Included the gray region of the LV-GLS cut-off value (absolute value $\leq 18$ ); B Not included the gray region of the LV-GLS cut-off value (absolute value $<16$ )

Table 4 Multivariate analysis for detecting independent predictors of impaired left ventricular global longitudinal strain (absolute LVGLS $\leq 18)$

\begin{tabular}{|c|c|c|c|c|c|}
\hline Variables & B & SE & Wald & OR $(95 \% \mathrm{Cl})$ & $P$ \\
\hline Age & -0.072 & 0.036 & 4.025 & $0.931(0.868-0.998)$ & 0.045 \\
\hline Gender & 1.265 & 0.728 & 3.022 & $\begin{array}{l}3.543(0.851- \\
14.748)\end{array}$ & 0.082 \\
\hline HT & -0.846 & 0.764 & 1.225 & $0.429(0.096-1.92)$ & 0.268 \\
\hline Smoking & -0.24 & 0.654 & 0.134 & $0.787(0.218-2.835)$ & 0.714 \\
\hline Asthma & -1.278 & 0.949 & 1.813 & $0.279(0.043-1.79)$ & 0.178 \\
\hline BMI & -0.053 & 0.059 & 0.792 & $0.949(0.845-1.065)$ & 0.373 \\
\hline GFR & -0.002 & 0.025 & 0.006 & $0.998(0.95-1.048)$ & 0.937 \\
\hline LDL & -0.006 & 0.008 & 0.577 & $0.994(0.979-1.01)$ & 0.447 \\
\hline CRP & 0.057 & 0.045 & 1.553 & $1.058(0.968-1.157)$ & 0.213 \\
\hline $\mathrm{Hg}$ & -0.15 & 0.237 & 0.402 & $0.86(0.541-1.369)$ & 0.526 \\
\hline E/E' & 0.133 & 0.16 & 0.689 & $1.142(0.834-1.563)$ & 0.407 \\
\hline DD & -0.106 & 0.624 & 0.029 & $0.899(0.265-3.053)$ & 0.865 \\
\hline $\begin{array}{l}\text { Recover- } \\
\text { ing from } \\
\text { COVID-19 }\end{array}$ & -2.121 & 0.625 & 11.51 & $0.12(0.035-0.408)$ & 0.001 \\
\hline
\end{tabular}

$C R P$ C-reactive protein, $E$ Peak early mitral filling velocity, $E$ ' Peak early diastolic myocardial velocity, GFR Glomerular filtration rate, $H g$ Hemoglobin, HT Hypertension, IVS Interventricular septum, $L V$ $E F$ Left ventricular ejection fraction, $L D L-C$ Low density lipoprotein cholesterol

dependent) could expect to indicate normal LV longitudinal systolic function [24].

In a single-center retrospective cohort study, Huang et al. [25] investigated 26 patients recovered from COVID-19. The study populations consisted of patients with no evidence of cardiac involvement during their initial treatment, which described chest discomfort or palpitations and other nonspecific cardiac symptoms one to three months after discharge (median 47 days). The authors reported 54\% of participants with evidence of myocardial edema using T2-weighted MRI. In a cohort of 100 unselected patients of recently recovered COVID-19 patients (median 47 days), Puntmann et al. [9]; found that $78 \%$ of patients had cardiac involvement, and $60 \%$ of patients had ongoing myocardial inflammation on MRI. Patients in the study consisted of hospitalized, outpatient, and patient groups with cardiovascular risk factors, and several patients had new or persistent symptoms. Li et al. [26] recently published a report of 218 patients hospitalized for COVID-19, including 52 critically and 166 non-critically ill patients. Their study, where they considered absolute value LV-LGS > 21 as the optimal cut-off, reported that the cardiac TnI increase was $10.8 \%$, the LVEF decrease was $22 \%$. In comparison, the deterioration in LV-GLS was $82 \%$. As a result, the authors suggested that most hospitalized COVID19 patients developed subclinical LV dysfunction detected by LV-GLS, despite preserved LVEF and normal levels of cTnI. They also reported that impaired LV-GLS (absolute value $<21$ ) was more common in critically ill patients $(98 \%)$ than in noncritical patients (78.3\%). In our study, although both were statistically significantly higher in patients who recently recovered from COVID-19 compared to the control group ( $p=0.02, p=0.04$, respectively); including the gray zone of the cut-off value (absolute value $\leq 18$ ), the proportion of patients with LV-GLS deterioration was $42.9 \%$; when did not include the gray zone (absolute value $<16$ ) it was $5.7 \%$. Our study determined LV-GLS cut-off values according to the latest detailed reports and the latest expert consensus mentioned above. The patient population consisted of low-risk asymptomatic outpatients. In addition, unlike many other studies, our study included a control group comprised of age- and sex-matched healthy subjects. These differences may explain the low percentage of left ventricular 
subclinical dysfunction in this study compared to the previous studies.

In our study, right ventricular (RV) function was evaluated with TAPSE and tricuspid lateral annular systolic velocity (S') which are practical, reliable, sensitive, and reproducible methods for initial diagnosis and follow-up of RV dysfunction. Although $S^{\prime}$ values were statistically significantly higher in the Covid 19 group, both $\mathrm{S}^{\prime}$ and TAPSE were within normal limits in both groups $(>9.5 \mathrm{~cm} / \mathrm{s}$, and $>16 \mathrm{~mm}$, respectively) [27]. In a recent study on patients hospitalized for Covid 19, as in our study, LV-GLS was impaired in the Covid-19 group, while LV structure, diastolic function, and right ventricular function were not different between groups [28]. In a recent MRI study conducted in symptomatic patients who recovered from Covid 19 , involvement was reported especially in the basal and midLV segments [25]. Furthermore, in numerous patient series and case reports a similar pattern of reduced LV basal strain was described in various forms of myocarditis [29, 30]. In an MRI study of patients with infiltrative cardiomyopathy, the researchers attributed this basal LV segment involvement to being the region of greatest activity throughout the cardiac cycle, and thus more susceptible to junctional stresses [31] Another possible mechanism may be a higher expression of angiotensin-converting enzyme 2 (ACE-2), the receptor of SARS-CoV-2, in epicardial adipose tissue (EAT), which is more prominent in the atrioventricular groove closer to the LV basal segments [32, 33].

The exact mechanism of COVID-19 related cardiac involvement is not yet precise. However, direct myocardial damage, myocardial disruption at the tissue level, myocarditis, an imbalance between the oxygen supply and demand of the myocardium, increased cardiac oxidative stress, the tendency to thrombosis due to systemic inflammation, stent thrombosis, inflammatory plaque rupture, and ACE-2 receptor-mediated systemic endothelitis are the most commonly proposed mechanisms [34-37]. Although more sensitive markers did not use in our study, CRP values, which the classic inflammation marker, were not different in healthy controls and COVID-19 groups. Moreover, all participants of our research consisted of asymptomatic patients with normal ECG and cardiac markers. Among these mechanisms, myocardial disruption at the tissue level or cardiomyocyte oxidative stress previously shown to be associated with impaired LV-GLS [37] seems to be the most logical explanation for LV-GLS deterioration in our patient group.

\section{Limitations}

Our study has several limitations that should address. The results did not validate for use in pediatric patients $(<18$ years of age) and those over 80 years of age. LV-GLS does not have a clear cut-off value and has a wide range of expected values due to vendor variability (type and model) of speckle tracking analysis. According to the absolute cutoff value $>18$, although values of LV-GLS were statistically significantly lower in the COVID-19 group, it was within the normal range in both groups. However, in a large metaanalysis of 2597 subjects from 24 studies, the published threshold of the mean absolute value of LV-GLS in healthy patients with preserved left ventricle function was 19.7 (95\% CI, 20.4-18.9) [38]. Moreover, although the absolute cut-off value was set as $>18$ in the last EACVI consensus report mentioned above, this cut-off value was $>20$ in the table of advanced echocardiographic parameters published in the report [21]. When these cut-off values were considered a reference, in our study, the control group had regular $(20.07 \pm 2.19)$, and the COVID-19 group had impaired LV-GLS values $(19.17 \pm 2.65)$. In addition, our study was a cross-sectional single-center pilot study carried out on a relatively small patient population. Finally, the primary purpose of our study was not to elucidate the etiopathogenesis of cardiac involvement of COVID-19, and we used only CRP to detect inflammation in our study. However, examining more sensitive inflammatory markers such as highly sensitive CRP (hs-CRP), tumor necrosis factor (TNF), or inflammatory cytokines could have contributed to interpreting the subclinical left ventricular dysfunction mechanism.

\section{Conclusions}

As a result of all these findings, our study suggests that COVID-19 may cause subclinical LV dysfunction detected by LV-GLS in the early recovery period even in a patient population at low cardiac risk, symptom-free, and recovered with home quarantine. Therefore, the cardiovascular followup of these patients may be more important than thought. More extensive studies and long-term follow-up is needed to confirm our finds and to predicting future cardiac events. Although there was no significant correlation between LVGLS and time from the initial COVID-19 diagnosis in our patient population in the first three mounts interval, we planned to re-evaluate the patients' 6-month and 1-year follow-up data.

Funding None.

\section{Declarations}

Conflict of interest All the authors declare no conflict of interest. 


\section{References}

1. Zhu N, Zhang D, Wang W, Li X, Yang B, Song J et al (2020) A novel coronavirus from patients with pneumonia in china, 2019. N Engl J Med 382:727-733

2. Clerkin KJ, Fried JA, Raikhelkar J, Sayer G, Griffin JM, Masoumi A et al (2020) Covid-19 and cardiovascular disease. Circulation 141:1648-1655

3. Hendren NS, Drazner MH, Bozkurt B, Cooper LT Jr (2020) Description and proposed management of the acute covid-19 cardiovascular syndrome. Circulation 141:1903-1914

4. Wu Z, McGoogan JM (2020) Characteristics of and important lessons from the coronavirus disease 2019 (covid-19) outbreak in china: Summary of a report of 72314 cases from the chinese center for disease control and prevention. JAMA 323:1239-1242

5. Yang J, Zheng Y, Gou X, Pu K, Chen Z, Guo Q et al (2020) Prevalence of comorbidities and its effects in patients infected with sars-cov-2: a systematic review and meta-analysis. Int J Infect Dis 94:91-95

6. Li B, Yang J, Zhao F, Zhi L, Wang X, Liu L et al (2020) Prevalence and impact of cardiovascular metabolic diseases on covid-19 in china. Clin Res Cardiol 109:531-538

7. Lippi G, Plebani M (2020) Laboratory abnormalities in patients with covid-2019 infection. Clin Chem Lab Med 58:1131-1134

8. Chapman AR, Bularga A, Mills NL (2020) High-sensitivity cardiac troponin can be an ally in the fight against covid-19. Circulation 141:1733-1735

9. Puntmann VO, Carerj ML, Wieters I, Fahim M, Arendt C, Hoffmann J et al (2020) Outcomes of cardiovascular magnetic resonance imaging in patients recently recovered from coronavirus disease 2019 (covid-19). JAMA Cardiol 5:1265-1273

10. Shmueli H, Shah M, Ebinger JE, Nguyen LC, Chernomordik F, Flint $\mathrm{N}$ et al (2021) Left ventricular global longitudinal strain in identifying subclinical myocardial dysfunction among patients hospitalized with covid-19. Int J Cardiol Heart Vasc 32:100719

11. Smiseth OA, Torp H, Opdahl A, Haugaa KH, Urheim S (2016) Myocardial strain imaging: how useful is it in clinical decision making? Eur Heart J 37:1196-1207

12. Yingchoncharoen T, Gibby C, Rodriguez LL, Grimm RA, Marwick TH (2012) Association of myocardial deformation with outcome in asymptomatic aortic stenosis with normal ejection fraction. Circ Cardiovasc Imaging 5:719-725

13. Conroy RM, Pyörälä K, Fitzgerald AP, Sans S, Menotti A, De Backer $G$ et al (2003) Estimation of ten-year risk of fatal cardiovascular disease in europe: the score project. Eur Heart J 24:987-1003

14. Williams B, Mancia G, Spiering W, Agabiti Rosei E, Azizi M, Burnier M et al (2018) esc/esh guidelines for the management of arterial hypertension: the task force for the management of arterial hypertension of the european society of cardiology (esc) and the european society of hypertension (esh). Eur heart j 39:3021-3104

15. Cockcroft DW, Gault MH (1976) Prediction of creatinine clearance from serum creatinine. Nephron 16:31-41

16. Lang RM, Badano LP, Mor-Avi V, Afilalo J, Armstrong A, Ernande L et al (2015) Recommendations for cardiac chamber quantification by echocardiography in adults: an update from the american society of echocardiography and the european association of cardiovascular imaging. J Am Soc Echocardiogr 28:1-39. e14

17. Nagueh SF, Smiseth OA, Appleton CP, Byrd BF 3rd, Dokainish H, Edvardsen $T$ et al (2016) Recommendations for the evaluation of left ventricular diastolic function by echocardiography: an update from the american society of echocardiography and the european association of cardiovascular imaging. J Am Soc Echocardiogr 29:277-314
18. Mor-Avi V, Lang RM, Badano LP, Belohlavek M, Cardim NM, Derumeaux G et al (2011) Current and evolving echocardiographic techniques for the quantitative evaluation of cardiac mechanics: Ase/eae consensus statement on methodology and indications endorsed by the japanese society of echocardiography. Eur J Echocardiogr 12:167-205

19. Thomas JD, Badano LP (2013) Eacvi-ase-industry initiative to standardize deformation imaging: a brief update from the cochairs. Eur Heart J Cardiovasc Imaging 14:1039-1040

20. Kalaycıŏglu E, Gökdeniz T, Aykan A, Hatem E, Gürsoy MO, Ören A et al (2014) Osteoprotegerin is associated with subclinical left ventricular systolic dysfunction in diabetic hypertensive patients: a speckle tracking study. Can J Cardiol 30:1529-1534

21. Biering-Sørensen T, Biering-Sørensen SR, Olsen FJ, Sengel $\varnothing v$ M, Jørgensen PG, Mogelvang R et al (2017) Global longitudinal strain by echocardiography predicts long-term risk of cardiovascular morbidity and mortality in a low-risk general population: The copenhagen city heart study. Circ Cardiovasc Imaging 10:e005521

22. Yang H, Wright L, Negishi T, Negishi K, Liu J, Marwick TH (2018) Research to practice: assessment of left ventricular global longitudinal strain for surveillance of cancer chemotherapeutic-related cardiac dysfunction. JACC Cardiovasc Imaging 11:1196-1201

23. Liu JE, Barac A, Thavendiranathan P, Scherrer-Crosbie M (2020) Strain imaging in cardio-oncology. JACC CardioOncology. 2:677-689

24. Galderisi M, Cosyns B, Edvardsen T, Cardim N, Delgado V, Di Salvo G et al (2017) Standardization of adult transthoracic echocardiography reporting in agreement with recent chamber quantification, diastolic function, and heart valve disease recommendations: an expert consensus document of the european association of cardiovascular imaging. Eur Heart J Cardiovasc Imaging 18:1301-1310

25. Huang L, Zhao P, Tang D, Zhu T, Han R, Zhan C et al (2020) Cardiac involvement in patients recovered from covid-2019 identified using magnetic resonance imaging. JACC Cardiovasc Imaging 13:2330-2339

26. Li R, Wang H, Ma F, Cui GL, Peng LY, Li CZ et al (2021) Widespread myocardial dysfunction in covid-19 patients detected by myocardial strain imaging using 2-d speckle-tracking echocardiography. Acta Pharmacologica Sinica. https://doi.org/10.1038/ s41401-020-00595-z

27. Rudski LG, Lai WW, Afilalo J, Hua L, Handschumacher MD, Chandrasekaran K et al (2010) Guidelines for the echocardiographic assessment of the right heart in adults: a report from the american society of echocardiography endorsed by the european association of echocardiography, a registered branch of the european society of cardiology, and the canadian society of echocardiography. J Am Soc Echocardiogr. 23:685-713

28. Goerlich E, Gilotra NA, Minhas AS, Bavaro N, Hays AG, Cingolani OH (2021) Prominent longitudinal strain reduction of basal left ventricular segments in patients with coronavirus disease- 19 . J Cardiac Fail 27:100-104

29. Ha SJ, Woo JS, Kwon SH, Oh CH, Kim KS, Bae JH et al (2013) Acute regional myocarditis with normal ventricular wall motion diagnosed by two-dimensional speckle tracking imaging. Korean J Intern Med 28:732-735

30. Hawwa N, Popovic ZB, Isma'eel HA (2015) Discordant electrocardiogram left ventricular wall thickness and strain findings in influenza myocarditis. Echocardiography (Mount Kisco, NY). 32:1880-1884

31. Deva DP, Hanneman K, Li Q, Ng MY, Wasim S, Morel C et al (2016) Cardiovascular magnetic resonance demonstration of the spectrum of morphological phenotypes and patterns of myocardial scarring in anderson-fabry disease. J Cardiovasc Magn Reson 18:14 
32. Abbara S, Desai JC, Cury RC, Butler J, Nieman K, Reddy V (2006) Mapping epicardial fat with multi-detector computed tomography to facilitate percutaneous transepicardial arrhythmia ablation. Eur J Radiol 57:417-422

33. Capotosto L, Nguyen BL, Ciardi MR, Mastroianni C, Vitarelli A (2020) Heart, covid-19, and echocardiography. Echocardiography (Mount Kisco, N.Y.) 37:1454-1464

34. Chen L, Li X, Chen M, Feng Y, Xiong C (2020) The ace2 expression in human heart indicates new potential mechanism of heart injury among patients infected with sars-cov-2. Cardiovasc Res 116:1097-1100

35. Varga Z, Flammer AJ, Steiger P, Haberecker M, Andermatt R, Zinkernagel AS et al (2020) Endothelial cell infection and endotheliitis in covid-19. Lancet (London, England) 395:1417-1418
36. Xu Z, Shi L, Wang Y, Zhang J, Huang L, Zhang C et al (2020) Pathological findings of covid-19 associated with acute respiratory distress syndrome. Lancet Respir Med 8:420-422

37. Haileselassie B, Su E, Pozios I, Niño DF, Liu H, Lu DY et al (2017) Myocardial oxidative stress correlates with left ventricular dysfunction on strain echocardiography in a rodent model of sepsis. Intensive Care Med Exp 5:21

38. Yingchoncharoen T, Agarwal S, Popović ZB, Marwick TH (2013) Normal ranges of left ventricular strain: a meta-analysis. J Am Soc Echocardiogr 26:185-191

Publisher's Note Springer Nature remains neutral with regard to jurisdictional claims in published maps and institutional affiliations. 